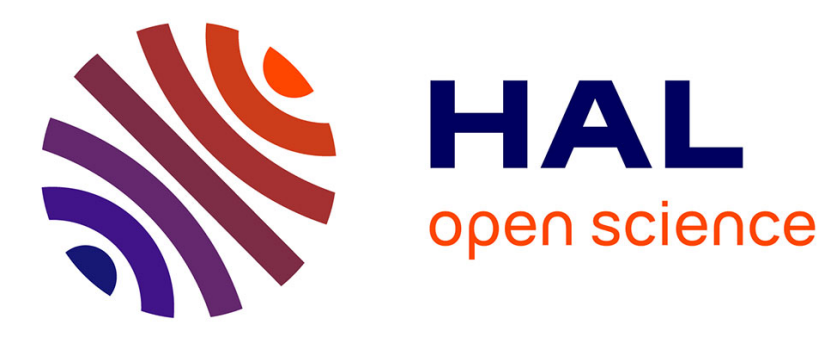

\title{
Spatially variant dimensionality reduction for the visualization of multi/hyperspectral images
}

\author{
Steven Le Moan, Alamin Mansouri, Yvon Voisin, Jon Hardeberg
}

\section{To cite this version:}

Steven Le Moan, Alamin Mansouri, Yvon Voisin, Jon Hardeberg. Spatially variant dimensionality reduction for the visualization of multi/hyperspectral images. International Conference on Image Analysis and Recognition, Jun 2011, Burnaby, Canada. pp.375-384, 10.1007/978-3-642-21593-3_38 . hal-00637936

\section{HAL Id: hal-00637936 https://u-bourgogne.hal.science/hal-00637936}

Submitted on 3 Nov 2011

HAL is a multi-disciplinary open access archive for the deposit and dissemination of scientific research documents, whether they are published or not. The documents may come from teaching and research institutions in France or abroad, or from public or private research centers.
L'archive ouverte pluridisciplinaire HAL, est destinée au dépôt et à la diffusion de documents scientifiques de niveau recherche, publiés ou non, émanant des établissements d'enseignement et de recherche français ou étrangers, des laboratoires publics ou privés. 


\title{
Spatially variant dimensionality reduction for the visualization of multi/hyperspectral images
}

\author{
Steven Le Moan ${ }^{1,2}$, Alamin Mansouri ${ }^{1}$, Yvon Voisin ${ }^{1}$, Jon Y. Hardeberg ${ }^{2}$ \\ ${ }^{1}$ Le2i, Université de Bourgogne, Auxerre, France \\ ${ }^{2}$ Colorlab, Høgskolen i Gjøvik, Norway
}

\begin{abstract}
In this paper, we introduce a new approach for color visualization of multi/hyperspectral images. Unlike traditional methods, we propose to operate a local analysis instead of considering that all the pixels are part of the same population. It takes a segmentation map as an input and then achieves a dimensionality reduction adaptively inside each class of pixels. Moreover, in order to avoid unappealing discontinuities between regions, we propose to make use of a set of distance transform maps to weigh the mapping applied to each pixel with regard to its relative location with classes' centroids. Results on two hyperspectral datasets illustrate the efficiency of the proposed method.
\end{abstract}

\section{Introduction}

Spectral imagery consists of acquiring a scene at more than three different ranges of wavelengths, usually dozens. Since spectral display devices are yet rare, most of today's popular display hardware is based on the tri-stimulus paradigm [1]. Thus, in order to visualize spectral images, a dimensionality reduction step is required so that only three channels (Red, Green and Blue for example) can contain most of the visual information while easing interpretation by preserving natural colors and contrasts [2]. At this aim, many dimensionality reduction techniques have been applied to the task of visualizing spectral datasets, they are roughly divided into two categories: either they operate a transformation or a selection of spectral channels. Even though the latter family is a subset of the former one, they are based on two very different philosophies. Indeed, band selection aim at preserving the physical meaning of spectral channels by keeping them intact during the $N$-to-3 projection, whereas band transformation allows any combination of channels (even nonlinear) as a means to fuse information along the spectrum. Therefore, the choice between these two approaches is of course application-driven. Band transformation methods are, for instance, based on the use of Principal Components Analysis (PCA) [3,4], Color Matching Functions (CMF) [5,2] or Independent Components Analysis [6]. Band selection strategies involve the use of similarity criteria such as correlation [7], Mutual Information [8] or Orthogonal Subspace Projection [9]. All these methods are based on the assumption that all the pixels are part of the same population, i.e. they perform a global mapping. Scheunders [10] proposed to spatially divide 
the image into blocks in order to achieve local mappings by means of PCA and Neural Network-based techniques. Discontinuities between blocks are dealt with by adapting the mappings at a pixel level. Not only do we propose to extend Scheunders' approach from a greyscale to a color framework, we enhance it in two ways: by using a classification map so as to choose which visual features deserve a local contrast enhancement, and by introducing a weighing function allowing to balance not only the influence of global versus local mapping, but also the respective influences of the individual classes. We will first introduce the different steps of the proposed approach: classification map, distance transforms and weighing of dimensionality reduction functions. Results will then be presented and discussed before conclusion.

\section{Spatially variant dimensionality reduction}

In this section, we give details on the different elements involved in the procedure.

\subsection{Segmentation map}

The first step of the proposed technique is to obtain a spatial segmentation of the image. This can be achieved either manually or automatically, by means of classifiers such as the K-Means, or Support Vector Machines. The choice of such a method is considered outside the scope of this paper as long as it is application-dependent and that the following processings apply anyway.

Let then $\operatorname{Seg}_{K}(I)$ be a segmentation map of image $I$ containing $K$ classes. While traditional methods consider each spectral channel as a whole, the core idea of the spatially variant dimensionality reduction is to analyze sets of pixels independently. For instance, if one desires to enhance the contrast between a couple of specific objects, one must consider the corresponding set of pixels separately from the others, in order to obtain a more dedicated analysis. Therefore, the final segmentation map must be computed not in a way that similar pixels are clustered together, but so that each class contains objects that need to be "separated".

\subsection{Distance transform}

In order to locally adapt the dimensionality reduction so that no discontinuities occur between regions, we need to know, for each location in the image, the distance to the closest centroids of each class. The distance transform is a way to efficiently achieve such measurements. It applies on binary images and consists of computing for each pixel with value 0 (black), its distance to the closest one with value 1 (white). Therefore, we need a set of binary images containing, in white, all the centroids of the different connected components from class $C_{i}$ and all the other pixels in black. We obtain such distance transform maps as the ones depicted in Figure 1c and 1f. Eventually, for a pixel $p_{(x, y)}^{c_{i}}$ at spatial coordinates $(x, y)$, belonging to class $c_{i}$, we obtain the set of its respective distances to the 
other classes centroids $\mathbf{d}(x, y)=\left[d_{1}(x, y), \ldots, d_{K}(x, y)\right]^{T}$, including the distance to the closest centroid of its own class.

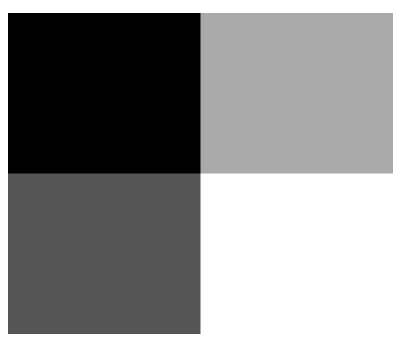

(a)

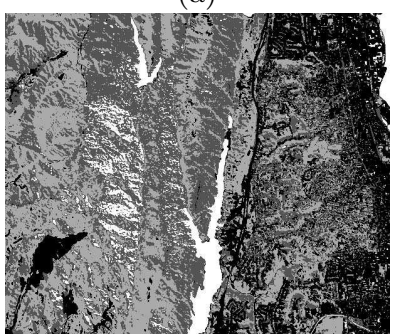

(d)

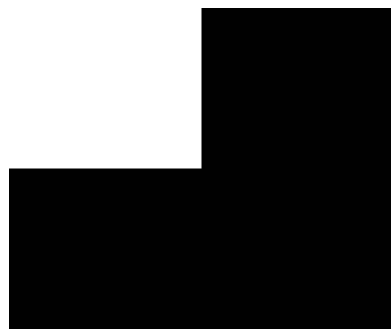

(b)

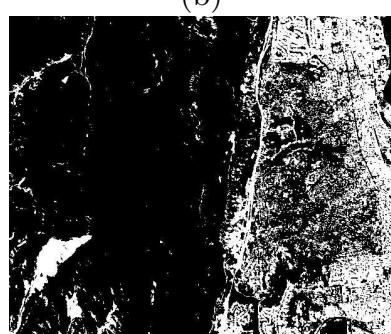

(e)

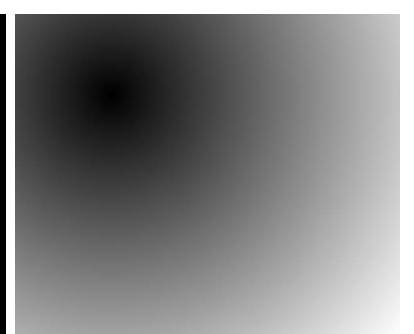

(c)

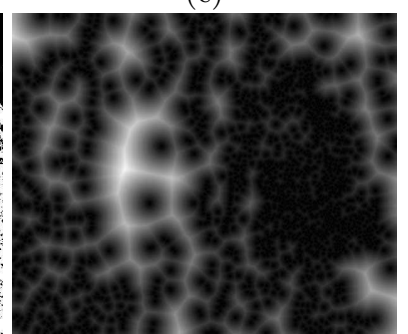

(f)

Fig. 1. Illustration of the distance transform applied on two possible segmentation maps for the "Jasper Ridge" dataset (see results section for full description). First column: segmentation maps (4 classes), Second column : class 1 isolated in white, Third column: the corresponding distance transforms. The first segmentation has been achieved manually, whereas the second one is the result of the K-means classifier.

\subsection{Weighing of dimensionality reduction functions}

Dimensionality Reduction (DR) is then performed in each class independently from the others so that we obtain as many sets of DR functions as there are classes. Moreover, a global mapping is also performed so as to be able to further balance between global and local mapping. What we refer to as a DR function is nothing more than a vector of coefficients used for fusing the spectral channels in order to obtain one of the three (Red, Green or Blue) primary bands. For instance, the Color Matching Functions (CMF) are such vectors.

Each pixel is then being affected with a set of weighted DR functions $D R^{R e d}(x, y)$, $D R^{\text {Green }}(x, y)$ and $D R^{\text {Blue }}(x, y)$ such that:

$$
D R^{R e d}(x, y)=\omega_{0} \times \mathbf{D R}_{0}^{R e d}+\left(1-\omega_{0}\right) \times \frac{\sum_{k \in(1 . . K)} \omega_{k} \times d_{k}(x, y) \times \mathbf{D} \mathbf{R}_{k}^{\text {Red }}}{\sum_{k \in 1 . . K} d_{k}(x, y)}
$$


with $\omega_{0}$ being the parameter allowing to balance between global and local mappings and $\omega=\left[\omega_{1}, \ldots, \omega_{K}\right]^{T}$, the vector of coefficients depicting the respective influences of the classes (its sum must be equal to one). The latter can be set manually or automatically, so that, for example, largest classes are given more weight. $\mathbf{D} \mathbf{R}_{0}^{R e d}$ is the global DR function and $\mathbf{D R}_{k}^{\text {Red }}, \forall i \in[1 . . K]$ are the local ones. Similar definitions apply of course for $D R^{\text {Green }}(x, y)$ and $D R^{\text {Blue }}(x, y)$.

\section{Experiments and results}

\subsection{Data sets}

For our experiments, we have used two hyperspectral datasets:

- "Jasper Ridge" is a well-known 220 bands image from the AVIRIS sensor [11]. We have used only a portion of the original dataset for the sake of clarity.

- "Norway" is a 160 bands remote sensing image, representing a urban area in the neighborhood of Oslo (Norway). It was acquired with the HySpex VNIR1600 sensor, developed by the Norsk Elektro Optikk company in Oslo. The sensor ranges from the early visible $(400 \mathrm{~nm})$ to the near infrared $(1000 \mathrm{~nm})$ with a spectral resolution of $3.7 \mathrm{~nm}$ [12].

As a pre-processing step, bands with average reflectance value below $2 \%$ and those with low correlation (below 0.8) with their neighboring bands have been removed, as suggested in [13].

\subsection{Dimensionality reduction techniques}

We have selected two dimensionality reduction techniques to illustrate the proposed approach.

- $P C A_{h s v}$ is the traditional Principal Components Analysis of which components are mapped to the HSV color space $(P C 1 \rightarrow V ; P C 2 \rightarrow S ; P C 3 \rightarrow H)$.

- $L P$ is a state-of-the-art band selection approached which has been proposed by $\mathrm{Du}$ et al. [9] and consists of progressively selecting bands by maximizing their respective orthogonality.

\subsection{Evaluation}

In order to evaluate the improvements by the proposed approach, we have, based on a K-means classification, selected 3 objects (or classes) of interest $\left(O b j_{1}, O b j_{2}\right.$ and $\mathrm{Obj}_{3}$ ) in each image and used the color difference metric CIE76 $\Delta E_{a b} *$ as a means to measure how contrasted they are. Obviously, the more they are contrasted, the more visual information we have. This metric has been applied on the objects' centroids (in the color space CIELAB). The objects of interest are depicted on Figure 2 for both datasets. For the spatially-variant dimensionality 


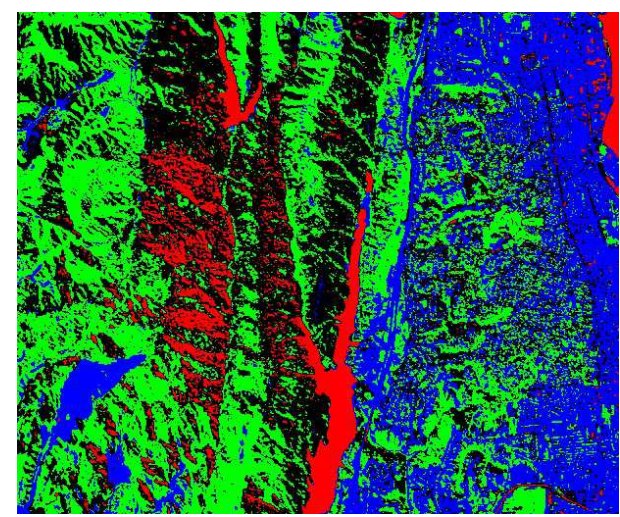

(a)

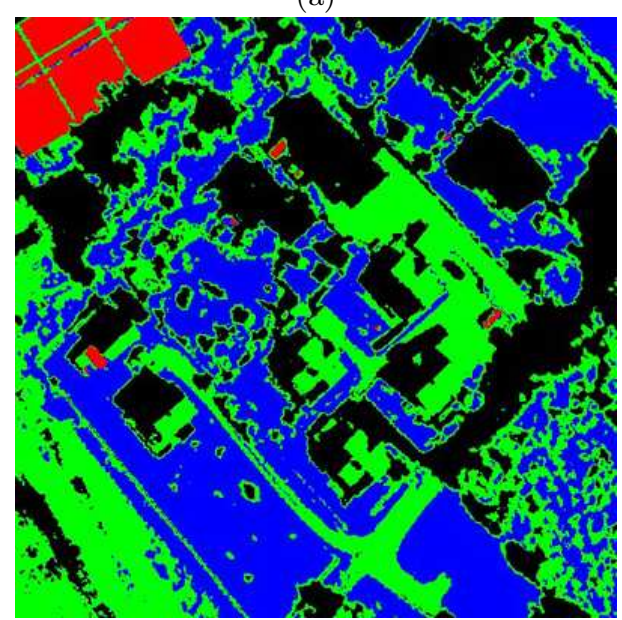

(c)

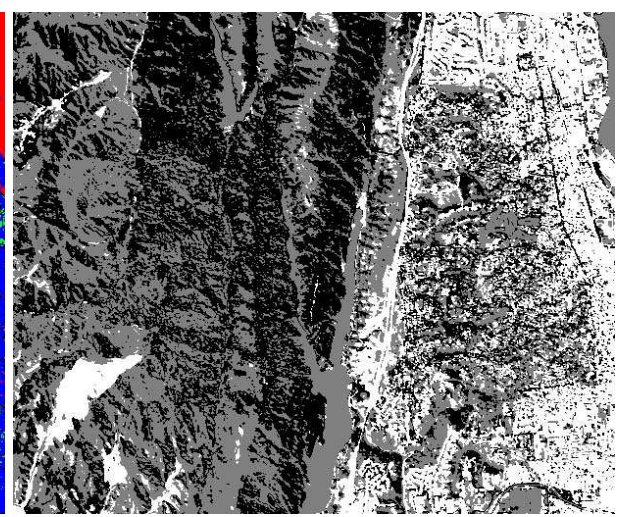

(b)

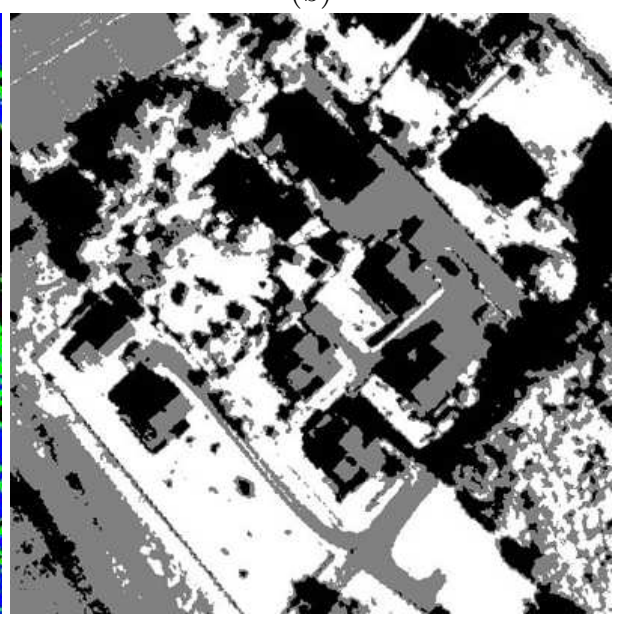

(d)

Fig. 2. Selected classes of interest $\left(O b j_{1}\right.$ in red, $O b j_{2}$ in green and $O b j_{3}$ in blue)

reduction, we have then used a segmentation map so that we obtain the three classes : $C_{1}=\left\{O b j_{1} \cup O b j_{2}\right\}, C_{2}=O b j_{3}$ and $C_{3}=\{$ rest of the pixels $\}$, as shown in Figures $2 \mathrm{~b}$ and $2 \mathrm{~d}$.

The object-separability metric will be referred to as Inter-Object Perceptual Separability (IOPS)

\subsection{Results}

Figures 3 and 4 depict respectively the resulting color composites by the global mappings $\left(\omega_{0}=1\right)$ and the local mapping $\left(\omega_{0}=0\right)$ without smoothing. Figure 5 depict the obtained color composites while Tables 1 and 2 give the results in terms of IOPS for the following configurations:

- Config 1: $\omega_{0}=0$ and $\omega=\left[\frac{1}{3}, \frac{1}{3}, \frac{1}{3}\right]$ 
- Config 2: $\omega_{0}=0$ and $\omega=[0.8,0.1,0.1]$

- Config 3: $\omega_{0}=0$ and $\omega=[0.1,0.8,0.1]$

- Config 4: $\omega_{0}=0.5$ and $\omega=\left[\frac{1}{3}, \frac{1}{3}, \frac{1}{3}\right]$

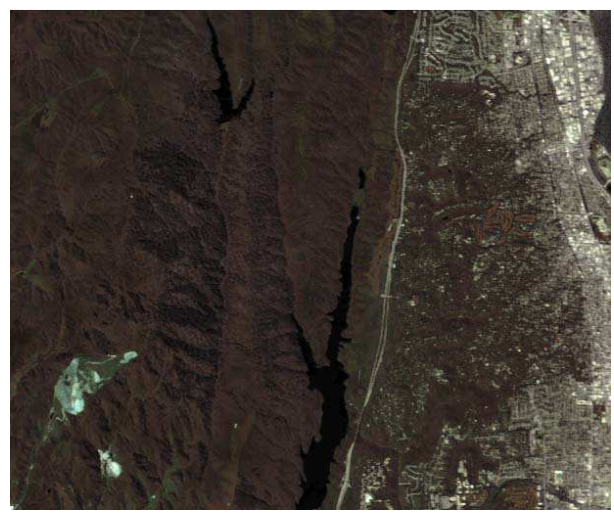

(a)

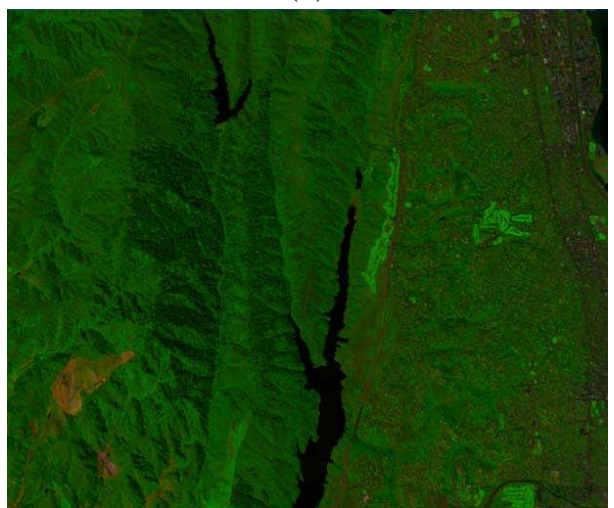

(c)

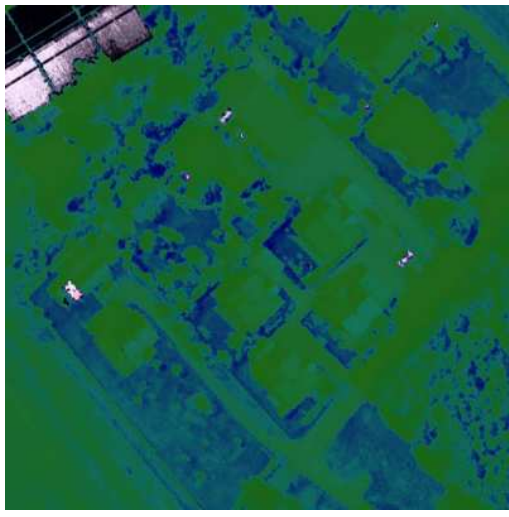

(b)

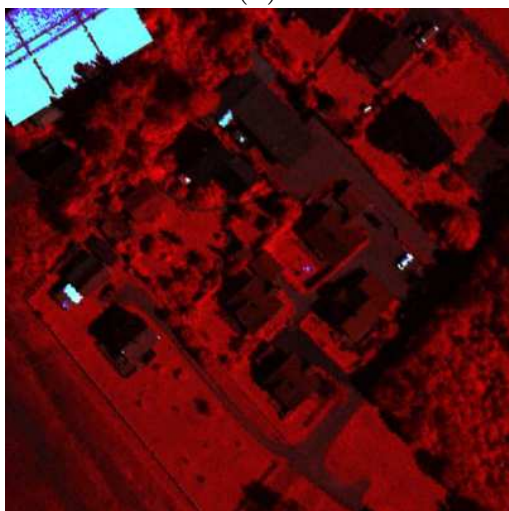

(d)

Fig. 3. Results obtained for $\omega_{0}=1$ (global only). First row: $P C A_{h s v}$, second row: LP-based band selection

\section{Comments on the results}

Based on the results presented in the previous section, we make the following remarks:

- The absence of weighing of the DR functions results in sharp discontinuities, as one can notice on Figures 4. Such artifacts are quite unappealing and thus do not allow for an efficient interpretation, hence the usefulness of the smoothing achieved by the weighing of the DR functions. 


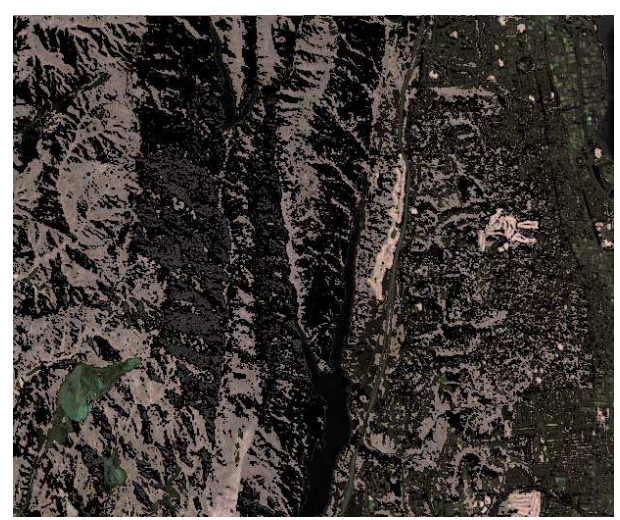

(a)

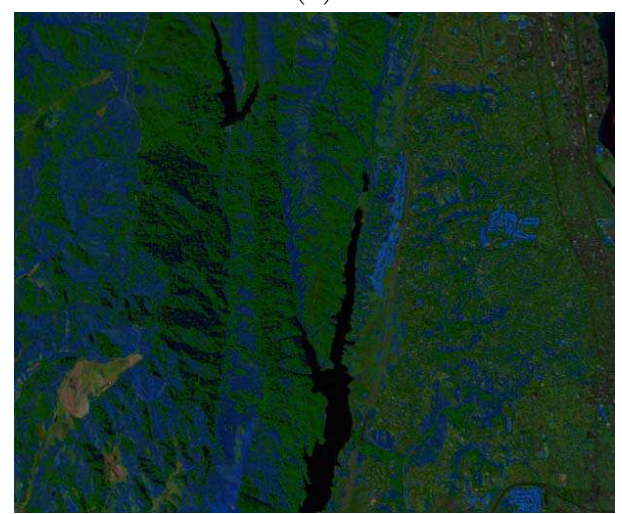

(c)

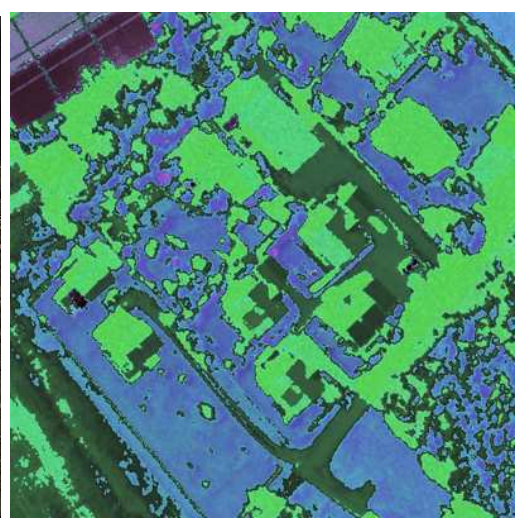

(b)

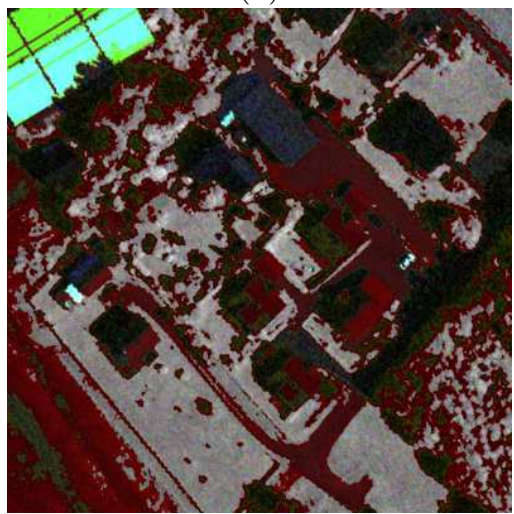

(d)

Fig. 4. Results obtained for $\omega_{0}=0$ and without weighing of the DR functions. First row: $P C A_{h s v}$, second row: LP-based band selection

- Overall, one can observe significant improvements from global to local techniques in the separation of Objects 1 and 2. On the other hand, separation between Objects 1 and 3 is better handled by the global approach. This comes from the fact that those three objects are given the same mapping in the global configuration, unlike in the local one.

- The second configuration always gives the best separation between Objects 1 and 2. This is due to the fact that this configuration gives more weight to the DR achieved in the class formed by these objects.

- The global DR allows for an overall better separation but local contrasts are not optimized, since better results are obtained from the first configuration, where all the classes are considered of equal influence. As a follow to that comment, the fourth configuration, which uses a 50-50 combination of global and local mappings gives the best compromise between both separations. 


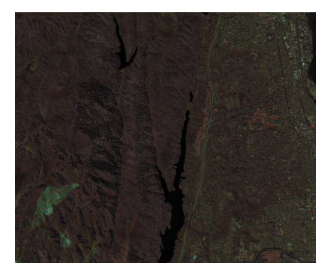

(a)

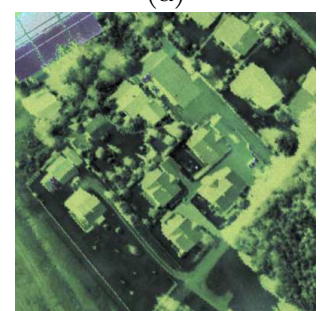

(e)

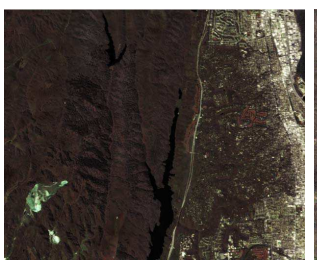

(b)

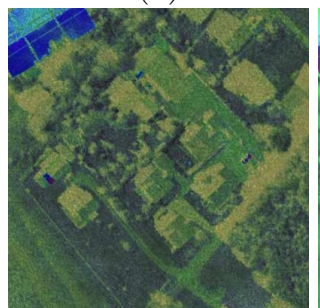

(f)

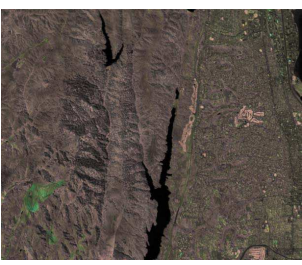

(c)

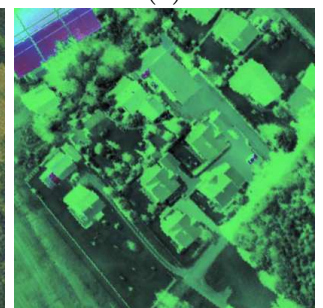

(g)

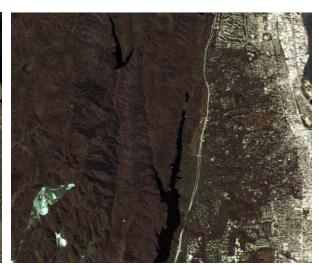

(d)

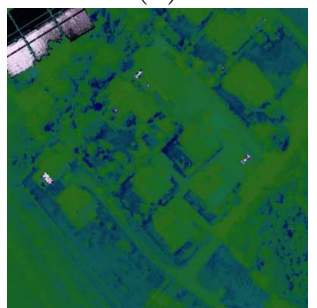

(h)

Fig. 5. Results obtained with the PCA-based dimensionality reduction, for all the configuration - First column: Config 1, second column: Config 2, third column: Config 3, fourth column: Config 4

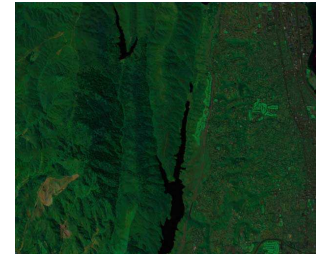

(a)

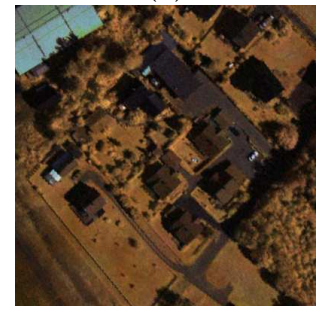

(e)

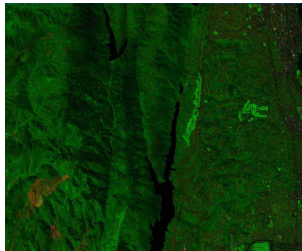

(b)

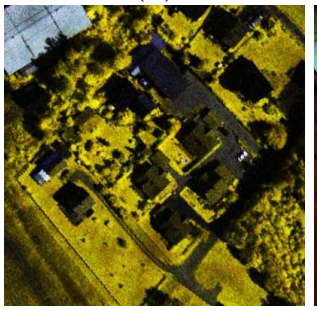

(f)

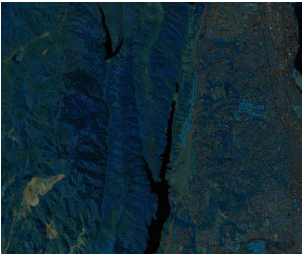

(c)

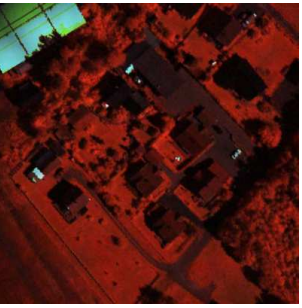

(g)

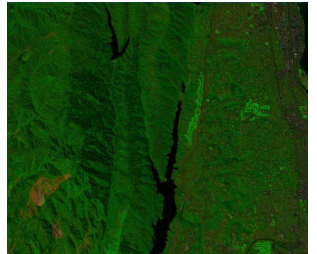

(d)

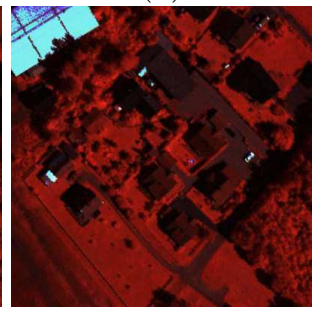

(h)

Fig. 6. Results obtained with the LP-based dimensionality reduction, for all the configuration - First column: Config 1, second column: Config 2, third column: Config 3, , fourth column: Config 4

\section{Conclusions}

An adaptive feature extraction algorithm has been presented, which takes into account dissimilarities between pixels by first clustering them and then conducting dimensionality reduction separately in each cluster. Preliminary results show 


\begin{tabular}{|c|l||c|c|}
\hline \multicolumn{2}{|c|}{} & $P C A_{h s v}$ & $L P$ \\
\hline \multirow{2}{*}{ Global } & $O b j_{1}$ vs. $O b j_{2}$ & 19.3 & 21.4 \\
& $O b j_{1}$ vs. $O b j_{3}$ & $\mathbf{3 1 . 0}$ & $\mathbf{1 2 . 2}$ \\
\hline \multirow{2}{*}{ Config 1 } & $O b j_{1}$ vs. $O b j_{2}$ & 35.7 & 34.3 \\
& $O b j_{1}$ vs. $O b j_{3}$ & 21.2 & 11.3 \\
\hline \multirow{2}{*}{ Config 2 } & $O b j_{1}$ vs. $O b j_{2}$ & $\mathbf{4 4 . 4}$ & $\mathbf{3 9 . 0}$ \\
& $O b j_{1}$ vs. $O b j_{3}$ & 26.1 & 11.0 \\
\hline \multirow{2}{*}{ Config 3 3} & $O b j_{1}$ vs. $O b j_{2}$ & 34.0 & 31.5 \\
& $O b j_{1}$ vs. $O b j_{3}$ & 21.3 & 10.3 \\
\hline \multirow{2}{*}{ Config 4 } & $O b j_{1}$ vs. $O b j_{2}$ & 27.3 & 30.9 \\
& $O b j_{1}$ vs. $O b j_{3}$ & 26.2 & 11.2 \\
\hline \multirow{2}{*}{ Perp }
\end{tabular}

Table 1. Inter-Object Perceptual Distance results for the "Jasper Ridge" image and for all the configurations considered

\begin{tabular}{|c|c|c|c|}
\hline & & $P C A_{h s v}$ & $L P$ \\
\hline \multirow{2}{*}{ Global } & $O b j_{1}$ vs. $O b j_{2}$ & 44.8 & 65.3 \\
\hline & $O b j_{1}$ vs. $O b j_{3}$ & 24.2 & 37.0 \\
\hline \multirow{2}{*}{ Config 1} & $O b j_{1}$ vs. $O b j_{2}$ & 56.0 & 68.0 \\
\hline & $O b j_{1}$ vs. $O b j_{3}$ & 21.3 & 27.7 \\
\hline \multirow{2}{*}{ Config 2} & $O b j_{1}$ vs. $O b j_{2}$ & 70.3 & $\overline{74.5}$ \\
\hline & $O b j_{1}$ vs. $O b j_{3}$ & 22.1 & 27.6 \\
\hline \multirow{2}{*}{ Config 3} & $O b j_{1}$ vs. $O b j_{2}$ & 60.1 & 71.2 \\
\hline & $O b j_{1}$ vs. $O b j_{3}$ & 24.0 & 28.6 \\
\hline \multirow{2}{*}{ Config 4} & $O b j_{1}$ vs. $O b j_{2}$ & 48.8 & $\overline{73.4}$ \\
\hline & $O b j_{1}$ vs. $O b j_{3}$ & 22.7 & 31.2 \\
\hline
\end{tabular}

Table 2. Inter-Object Perceptual Distance results for the "Norway" image and for all the configurations considered

an increasing amount of informative as well as perceptual content. The technique being obviously very sensitive to the pixel clustering conducted prior to dimensionality reduction, this step will be further investigated along with the influence of other feature extraction techniques as well as other distance metrics in order to draw a more complete evaluation of the spatially-variant dimensionality reduction.

\section{Acknowledgements}

The regional council of Burgundy supported this work.

\section{References}

1. Grassmann, H.: On the theory of compound colors. Phil. Mag 7 (1854) 254-64 
2. Jacobson, N., Gupta, M.: Design goals and solutions for display of hyperspectral images. IEEE Trans. on Geoscience and Remote Sensing 43 (2005) 2684-2692

3. Jia, X., Richards, J.: Segmented principal components transformation for efficient hyperspectral remote-sensing image display and classification. IEEE Trans. on Geoscience and Remote Sensing 37 (1999) 538-542

4. Tyo, J., Konsolakis, A., Diersen, D., Olsen, R.: Principal-components-based display strategy for spectral imagery. IEEE Trans. on Geoscience and Remote Sensing 41 (2003) 708-718

5. Poldera, G., van der Heijdena, G.: Visualization of spectral images. In: Proc. SPIE. Volume 4553. (2001) 133

6. Hyvärinen, A., Oja, E.: Independent component analysis: algorithms and applications. Neural networks 13 (2000) 411-430

7. Chang, C., Du, Q., Sun, T., Althouse, M.: A joint band prioritization and banddecorrelation approach to band selection for hyperspectral image classification. IEEE Trans. on Geoscience and Remote Sensing 37 (1999) 2631-2641

8. Guo, B., Damper, R., Gunn, S., Nelson, J.: A fast separability-based featureselection method for high-dimensional remotely sensed image classification. Pattern Recognition 41 (2008) 1670-1679

9. Du, Q., Yang, H.: Similarity-based unsupervised band selection for hyperspectral image analysis. IEEE Geoscience and Remote Sensing Letters 5 (2008) 564-568

10. Scheunders, P.: Multispectral image fusion using local mapping techniques. In: International conference on pattern recognition. Volume 15. (2000) 311-314

11. [online]: http://aviris.jpl.nasa.gov/html/aviris.freedata.html (last check : Nov. 11, 2010)

12. [online]: http://www.neo.no/hyspex/ (last check : Nov. 11, 2010)

13. Cai, S., Du, Q., Moorhead, R.: Hyperspectral imagery visualization using double layers. IEEE Trans. on Geoscience and Remote Sensing 45 (2007) 3028-3036 